\title{
Do Fixed Dose Combinations Play an Important Role in the Management of Coexistent Type Two Diabetes Mellitus and Hypertension?
}

\author{
Nisharani Ranpise*, Preeti Jamkar, Harshada Langote \\ Department of Pharmaceutics Sinhgad College of Pharmacy, Vadgaon (Bk.), Pune-411041 Maharashtra, INDIA.
}

\begin{abstract}
The global burden of coexistent Type Two Diabetes Mellitus (TTDM) and hypertension is rising and it is emerging as a severe threat to world health and well being. This twin epidemic of coexistent TTDM and hypertension exposes patients to severe co-morbidities like cardiovascular diseases, lower limb amputations, diabetic nephropathy, diabetic retinopathy if not treated in time and with appropriate regimen. Guidelines established by various international organizations suggest multiple drug therapy for the safe and effective management of coexistent TTDM and hypertension. Such a polypharmacy with complex drug regimen leads to increased pill burden and often reflects into decreased patient compliance. This poor adherence to prescribed regimen, then gets translated into worsening of conditions and increases the hospitalization and all cause mortality to a significant extent. Use of FDCwill be one of the promising solution in the treatment of coexistent TTDM and hypertension where patients have to take 4-5 tablets on an average per day and thus show poor compliance to therapy. Use of FDC leads to the decrease in pill burden and thus helps in improving patient compliance. Along with these facts formulations of FDC also leads to simplification of complex drug regimen, synergistic effect produced by dugs in combination and reduction in cost of product. Present review describes the role of FDC in the treatment of coexistent TTDM and hypertension.
\end{abstract}

Key words: Coexistent type two diabetes mellitus and hypertension, Treatment for coexistent type two diabetes mellitus and hypertension, Fixed dose combination.

\section{INTRODUCTION}

The increasing global burden of coexistent Type Two Diabetes Mellitus (TTDM) and hypertension isemerging as a severe threat to world health and well being.

Diabetes Mellitus (DM) is characterized by hyperglycemia; altered metabolism of lipids, carbohydrates and proteins; and an increased risk of complications from vascular disease. Type $1 \mathrm{DM}$ is also characterized by an extensive and selective loss of pancreatic $\beta$ cells and a state of absolute insulin deficiency. In TTDM approximately 50\% reduction in $\beta$-cell mass is observed resulting in a profound defect in first-phase insulin secretion and insulin resistance. ${ }^{1}$
The American Diabetes Association (ADA) criteria for the diagnosis of TTDM include symptoms like polyuria, polydipsia including unexplained weight loss and a random plasma glucose concentration $>200 \mathrm{mg} / \mathrm{dL}$ (11.1 mM)or fasting plasma glucose concentration $>126 \mathrm{mg} / \mathrm{dL}(7 \mathrm{mM})$, or $\mathrm{aHb}$ $\mathrm{A} 1 \mathrm{C} \geq 6.5 \%$. Prevalence of this chronic disorder is increasing worldwide due to sedentary lifestyle which leads to increased obesity and less physical activity. ${ }^{2}$ Upto 2012 diabetes has affected 371 million people in the world and this number may reach to 439 million by 2030 . Around $36 \%$ of the anticipated absolute global increase in number of diabetes patients is projected to happen alone in India in coming 18-19 years. ${ }^{3}$
Submission Date :07.09.2014 Revision Date $\quad: 09.12 .2014$ Accepted Date : :16.01.2014 DOI: 10.5530/ijper.49.3.4 Correspondence Address Dr.Nisharani Ranpise Professor and HOD of Pharmaceutics, Sinhgad College of Pharmacy, Vadgaon (Bk.), Pune-411041

Maharashtra, India Phoneno:+91-9822671472 E-mail:nisha_ranpise@ yahoo.com

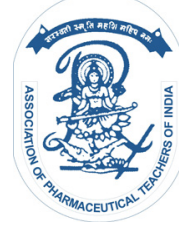

www.ijper.org 
Table 1: Commonly prescribed antihypertensive and oral hypoglycemic agents in treatment of co-existent TTDM and hypertension

\begin{tabular}{|c|c|}
\hline Category of Drug & Example \\
\hline \multicolumn{2}{|r|}{ Antihypertensive agent } \\
\hline ACE inhibitors & $\begin{array}{l}\text { Ramipri, Benazepril, Captopril, Enalapril, Fosinopril, Lisinopril, } \\
\text { Moexipril Perindopril, Quinapril, Trandolapril }\end{array}$ \\
\hline Beta blockers & Atenolol, Metoprolol, Nebivolol, Bisoprolol, Metoprolol, Timolol \\
\hline Angiotensin II antagonists & $\begin{array}{l}\text { Telmisartan, Olmesatan, Candesartan, Eprosartan, Irbesartan, } \\
\text { Losartan }\end{array}$ \\
\hline CCB (DHP) & Amlodipine, Felodipine, Isradipine, Nicardipine, Nifedipine \\
\hline CCB (non DHP) & Diltiazem, Verapamil \\
\hline HMG Co-A Reductaselnhibitors & Atorvastatin, Rosuvastatin, Pravastatin, Simvastatin \\
\hline Antiplatelet aggregatory agent & Aspirin, Clopidogrel \\
\hline \multicolumn{2}{|r|}{ Oral hypoglycemic agents } \\
\hline Biguanide & Metformin \\
\hline Sulfonylureas 2ndgeneration & Glyburide/glibenclamide, GlipizideGliclazide, Glimepiride \\
\hline Meglitinides (glinides) & Repaglinide, Nateglinide \\
\hline Thiazolidinediones & Pioglitazone, Rosiglitazone \\
\hline$\alpha$-Glucosidase inhibitors & Acarbose, Voglibose \\
\hline DPP-4 Inhibitors & Sitagliptin, Vildagliptin, Saxagliptin, Linagliptin \\
\hline
\end{tabular}

But the major concern for global health is that along with the TTDM prevalence of hypertension is also rising. In developed countries $60-65 \%$ prevalence is seen for the twin epidemic of this 'deadly combination'. Rarely TTDM and hypertension are seen isolated nowadays. This is because diabetic patients are two times more predisposed to have hypertension. ${ }^{4}$ Sometimes, hypertension may appear before in TTDM patients, but nephropathy and insulin sensitivity (resistance) are the major factors which contribute significantly and independently in the genesis of hypertension amongst diabetics. Raised insulin levels in diabetic patients promote sodium retention and along with the sodium glucose also gets reabsorbed. This sodium and fluid retention generates hypervolemia and hypertension. ${ }^{5}$ As a consequence of various biochemical phenomenon occurring in diabetic individuals such as increase in the activity of liver lipase which leads to lipid abnormalities like increase in VLDL levels, reduction in HDL cholesterol, hypertriglyceridaemia, generation of dense and short LDL particles, hypertension appears in diabetic individuals. $^{6}$

Propensity of developing cardiovascular diseases is also doubled in patients with co-occurrence of TTDM and hypertension. ${ }^{7}$ TTDM and hypertensionco-existence exposes patients to complications like microangiopathy (renal, retinal and neural), macroangiopathy (atherosclerosis) and lower limb amputations. ${ }^{8-13}$ Diabetic nephropathy (DN) is usually the reason for the development of end stage renal diseases in hypertensive diabetic patients. These associated severe complications adversely affect quality of life of patients and also leads to increases in economical burden on patients.

Against this milieu of increasing co-occurrence of TTDM and hypertension worldwide, safe and effective treatment for the same must be discussed as a public health priority.

Guidelines established by various international organizations suggest multiple drug therapy for the safe and effective management of co-existent TTDM and hypertension. (Table 1) ADA recommends the use of metformin (if tolerated and not contraindicated), ACEI or $\mathrm{ARB}$, any statin along with aspirin (antiplatelet agent) as a first line therapy to maintain the targeted blood glucose level, essential blood pressure control and also to avoid further cardiovascular complications. In some chronic cases intensified therapy with addition of some more pharmacological agents (e.g.) sulphonylurea or GLP 1 agonist or CCB to the above mentioned regimen is required. ${ }^{2}$ Such a complex drug regimen leads to increased pill burden and often reflects into decreased patient compliance. This poor adherence to prescribed regimen, then gets translated into worsening of conditions and increase in hospitalization and all cause mortality to a significant extent. ${ }^{14-15}$

\section{GUIDELINE RECOMMENDATIONS FOR MANAGEMENT OF COEXISTENT TTDM AND HYPERTENSION ${ }^{2,16-20}$}

The ADA recommends the use of metformin as a preferred initial therapy (if tolerated and not contraindicated). Insulin therapy comes into consideration 
in newly diagnosed patients with markedly symptomatic and/or elevated blood glucose levels or $\mathrm{Hb} \mathrm{A} 1 \mathrm{C}$ with or without other agents at the outset. If noninsulin monotherapy at the maximal tolerated dose does not achieve or maintain $\mathrm{Hb}$ A1C target over 3-6 months addition of second oral agent, GLP-1 receptor agonist, or insulin comes into consideration. A guideline by the International Diabetes Federation (IDF) suggests that metformin should be used with caution if estimated glomerular filtration rate (eGFR) $<45 \mathrm{ml} / \mathrm{min} / 1.73 \mathrm{~m}^{2}$. When glucose control targets are not being achieved, addition of a sulfonylurea is recommended. Other options include adding metformin if not used first-line, an $\alpha$-glucosidase inhibitor, a Dipeptidyl Peptidase-4 (DPP-4) inhibitor or a thiazolidinedione.

The ADA recommends the use of ACEI or ARB as the drug of choice for persons with diabetes and hypertension. These guidelines further suggest that $\geq 2$ agents at maximal doses are usually required to achieve BP targets. It also says that administration $\geq 1$ agent should be done at bedtime.

The Seventh Report of the Joint National Committee on Prevention, Detection, Evaluation, and Treatment of High Blood Pressure (JNC 8) says that diuretics, ACEIs, BBs, ARBs, and CCBs are beneficial in the treatment of hypertension in both type 1 and TTDM and it is well proven with clinical trials. The question of which class of agent is superior for lowering BP is somewhat unanswered because the majority of diabetic patients will require two or more drugs to achieve BP control.

The use of ACEIs and ARBs may offer some advantages over other agents in some situations, but they should not be used together. CCBs should be avoided in diabetic patients with congestive heart failure. IDF guidelines suggest the use of $\beta$-adrenergic blockers in people with angina; $\beta$-adrenergic blockers and ACEIs in people with coronary artery disease; ACEIs or diuretics in those with heart failure; ACEIs plus low dose thiazide or thiazide-like diuretic (indapamide or chlorthalidone), or ACEIs plus CCB in people with cerebrovascular disease. Care should be taken with combined thiazide and $\beta$-adrenergic blockers because of risk of deterioration in metabolic control.Addition of further medications from a different class is done if targets are not reached on maximal doses of current medications, reviewing for adverse effects and likelyadherence problems as tablet numbers increase.

ADA suggests statins in lower-risk patients (no overt CVD, aged $<40 \mathrm{yrs}$ ) if LDL-C $>100 \mathrm{mg} / \mathrm{dL}$ or in the presence of multiple CVD risk factors. (Family history of CVD, hypertension, smoking, dyslipidemia, or albuminuria)
For primary prevention of coronary heart disease $75-162 \mathrm{mg} /$ day of aspirin is recommended in patients with type 1 and 2 diabetes at increased CVD risk (10-yr risk $>10 \%$ ) and $75-162 \mathrm{mg} /$ day of aspirin is recommended in patients with diabetes and history of CVD for secondary prevention by majority of guidelines.

\section{RATIONALE BEHIND SELECTION OF THERAPEUTIC AGENTS FOR TREATMENT OF COEXISTENT TTDM AND HYPERTENSION}

To provide a strong rationale for combination therapy in hypertension and TTDM extensive work has been done and still it is going on. Along with oral hypoglycemic agents guidelines suggest use of ARBs, ACEIs, statins, aspirin and other drug categories also to effectively control blood pressure and blood glucose levels which will also help in reducing microvascular and macrovascular complications associated with it. The rationale behind this multidrug therapy can exemplified as follows.

\section{ARBs and ACEls}

Angiotensin II causes an increase in serine phosphorylation of the insulin receptor, insulin receptor substrate 1, and phosphatidylinositol-3-kinase (PI3K), which leads to impairment in insulin signaling and this contributes to insulin resistance. Therefore, blocking of the RAAS system will prevent all these events and will help in reducing the insulin resistance. ${ }^{21,22}$ In diabetic or uremic serum, ARB and ACEI inhibit the formation of advanced glycation end products (AGE) previously implicated in the pathology of diabetic complications and atherosclerosis. The AGE inhibitory effect of ARB, unlike that of ACEI, is linked to a common core structure 5-(4-methylbiphenyl-2-yl)-1H-tetrazol. Thus, it is a class effect. By contrast, ACEI has no common core structure and it is not conclusive whether the AGE inhibitory effect of ACEI is a class effect. ARB thus constitute a unique class of therapeutic agents since they have property of inhibition of advanced glycation, oxidative metabolism and the chelation of transition metals which are absent in CCB and $\beta$ blockers. ${ }^{23}$

S. Yamagishia, M. Takeuchi hypothesize that due to its unique PPAR- $\gamma$-modulating activity, telmisartan will become a promising 'cardiometabolicsartan'. By acting as a partial agonist of PPAR $\gamma$ it influences the expression of PPAR $\gamma$ target genes involved in carbohydrate and lipid metabolism; and reduce glucose, insulin, and triglyceride levels in rats fed a high-fat, high-carbohydrate diet. Thus, it has the potential to target diabetes and cardiovascular diseases in hypertensive patients..$^{24,25}$ Through activation of PPAR $\delta$ dependant pathways telmisartan prevents adipogenesis in vivo and in vitro and leads to anti obesity effect which is neither shown by candesartan nor by losartan. This effect of telmisar- 
tan preventing weight gain and obesity may help in the treatment of diabetic hypertensive patients. ${ }^{26}$

Telmisartan and amlodipine were studied for their effect on metabolic parameters and blood pressure in type two diabetic hypertensive patients. This study revealed that telmisartan improvesthe metabolic profile better than amlodipine in patients of TTDM treated with rosiglitazone. ${ }^{21}$

Irbesartan and losartan also slows down the progression of glomerulopathy exhibiting renoprotective effect along with improved blood pressure control in TTDM patients. ${ }^{27,28}$

Elevated Urine Albumin Excretion (UAER) is a modifiable risk factor for renal and cardiovascular disease in type 2 diabetes. ARBs can reduce the rate of progression from microalbuminuria to macroalbuminuria and to ESRD (End Stage Renal Disease) in patients with type 2 diabetes. Valsartanlowered UAER more effectively than amlodipine in patients with type 2 diabetes and microalbuminuria independent of its BP lowering action. ${ }^{29}$ Similarly olmesartan also delays microalbuminurea in patients with TTDM. ${ }^{30}$

ONTARGET $^{\circledR}$ demonstrated that the ARBs and ACEI were equally effective in reducing the primary composite outcome of $\mathrm{CV}$ death, myocardial infarction, stroke or hospitalization due to heart failure (relative risk, 1.01; 95\% CI, 0.94-1.09), but that telmisartan was better tolerated than ramipril. ${ }^{31}$ Though ARBs offer the advantage of lack of cough and well tolerability as compared to ACEI, but still there are no strong evidences which will decide the superiority amongst these two agents. ${ }^{32,33}$

\section{Lipid management therapy}

People with TTDM and hypertension are at high risk of cardiovascular diseases. Lipoprotein abnormalities are manifested during the pre-diabetic stage in the development of frank diabetes, even earlier than insulin resistance and hyperglycemia, and it contributes substantially to the increased risk of macrovascular disease. The dyslipidemia in diabetic patients is characterized by elevated triglyceride levels and decreased HDL cholesterol levels. Qualitative changes in LDL cholesterol are prominent in patients with diabetes and tend to have a higher proportion of LDL particles that are smaller and denser which are more susceptible to oxidation and may thereby increase the risk of cardiovascular events ${ }^{34}$ To lower the blood lipid levels currently several pharmacological agents are available like HMG Co-A reductase inhibitors, fibrates, extended-acting nicotinic acid, concentrated omega-3 fatty acids, ezetimibe, bile acid binding resins etc. ${ }^{14}$
All patients with diabetes should be prescribed statin therapy to achieve a reduction in LDL cholesterol by at least $30-40 \%$, to a target goal of $<100 \mathrm{mg} / \mathrm{dl}(2.60$ $\mathrm{mmol} / \mathrm{l})$. This approach is supported by evidence showing that all diabetic patients are in the high risk category, and the majority of those considered at lower risk, mostly younger individuals, will attain a risk of $20 \%$ over a 20 -year period. ${ }^{35}$

Management with fibrates is indicated if serum triglyceride levels are raised and HDL cholesterol is low. Fibrates significantly reduce non-fatal MI (Myocardial Infarction) but have no significant effect on CVDor all-cause mortality, fatal MI or stroke, all of which are significantly reduced by statins. ${ }^{14}$ Myopathy and rhabdomyolysis are the major adverse reactions associated with fibrates. Gemfibrozil co-administration with statins leads to increased risk of myopathy. ${ }^{36}$

The evidence-base for other lipid-lowering medications (extended-acting nicotinic acid, concentrated omega-3 fatty acids, ezetimibe and bile acid binding resins) is weaker and there are very few quality outcomes studies. The use of these agents is generally reserved for uncontrolled hyperlipidaemia when taking first-line agents, or intolerance of these.

\section{Aspirin therapy}

Aspirin therapy is recommended in diabetic and hypertensive patients for primary prevention of cardiovascular disease. Aspirin imparts its primary antithrombotic effects through the inhibition of PGH-synthase/COX by the irreversible acetylation of a specific serine moiety (serine 530 of COX-1 and serine 516 of COX-2) ${ }^{11,12}$ and is ' 170 -fold more potent in inhibiting COX-1 than COX-2. $75-162 \mathrm{mg} /$ day of aspirin is recommended in patients with types 1 and 2 diabetes at increased CVD risk $(10$-yr risk $>10 \%){ }^{37}$

High sensitivity of aspirin towards platelet inhibition shows that a dose as low as $75 \mathrm{mg}$ of enteric-coated aspirin is just as effective as higher doses of either plain or enteric coated aspirin in inhibiting thromboxane synthesis. ${ }^{38}$ Aspirin significantly reduced cardiovascular events by $15 \%$ and myocardial infarction by $36 \%{ }^{39}$ Low-dose aspirin therapy reduces the risk of atherosclerotic events in type 2 diabetic patients with eGFR 60-89 $\mathrm{mL} / \mathrm{min} / 1.73 \mathrm{~m} .{ }^{40}$ Aspirin should not be prescribed to patients with aspirin allergy, bleeding tendency, anticoagulant therapy, recent gastrointestinal bleeding and clinically active hepatic disease. In such cases other antiplatelet agents should be used.

\section{$\beta$ Blockers}

$\beta$ blockers are proved to be promising for treatment of hypertension. But they are underused for treating 
hypertension in TTDM. The Glycemic Effect in Diabetes Mellitus: Carvedilol Metoprolol Comparison in Hypertensives (GEMINI) trial was the first randomized, double-blind, controlled clinical trial evaluating the effect of adding $\beta$-blockers to standard therapy with renin-angiotensin system (RAS) blockade to achieve BP control in patients with diabetes and hypertension. This trial revealed that carvedilol is beneficial in hypertensive diabetic patients with fewer unfavorable effects as carvedilol in the presence of RAS blockade did not affect glycemic control and improved some components ofthe metabolic syndrome relative to metoprolol in participants with DM and hypertension. ${ }^{41,42} \beta-1$ blockade might be expected to inhibit the harmful actions of sustained high noradrenalin activity and thus they are emerging as one of the promising treatment for hypertensive diabetic patients. ${ }^{43} \beta$ blockers are indicated in patients with TTDM after MI. $\beta$ Blockers have antiischemic, anti-arrhythmic and anti-rennin/angiotensin properties. $\beta$ blockers also tend to prolong coronary diastolic filling time; up regulate cardiac b1 receptors and inhibit stimulatory anti b1-receptor auto antibodies, augment atrial and brain naturetic peptide, lower plasma endothelin-1 levels (carvedilol), stimulate the endothelial L-arginine/nitric oxide pathway (vasodilatory betablockers such as nebivolol) and inhibit catecholamine induced cardiac necrosis (apoptosis), all of which are advantageous for patients. ${ }^{44}$ Use of highly $b_{1}$ selective agents should be done to avoid risk of bronchoconstriction and the inhibition of $b_{2}$ stimulant bronchodilation in sensitive subjects. ${ }^{45} \mathrm{~b}_{2}$ blockade is inappropriate for the treatment of hypertensive type II diabetics, who may be on insulin therapy, and a highly $\mathrm{b}_{1}$ selective betablocker would be the agent of choice. ${ }^{46}$

\section{CCBs and diuretics}

CCBs and diuretics can be used as second line or third line agents in the management of hypertension associated with TTDM.

Presence of L-type calcium channels only on afferent arterioles leads to elevation of glomerular pressure after inhibition while blockade of $\mathrm{N}$ type calcium channels leads to well-balanced dilation of both arterioles since they are located in sympathetic nerve endings, controlling both afferent and efferent arterioles. Thus clinidipinea $\mathrm{N}$ type CCB leads to reduction of glomerular pressure and urinary protein. Sympathetic suppression induced by clinidipine through the inhibition of $\mathrm{N}$-type calcium channels leads to reduced secretion of renin from periglomerularcells, which is further responsible for renoprotective effect. CCB, which inhibits L- and $\mathrm{N}$-type calcium channels may be useful for patients with hypertension and diabetes mellitus from its effects on lipid metabolism and renal function. ${ }^{47,48}$

A study carried out by Robert D. Toto and other scientist suggest that administration of FDCs of an ACEI with either an NDHP CCB or a DHP CCB can effectively reduce albuminuria in type 2 diabetics with hypertension and nephropathy. ${ }^{49}$ If patients blood pressure is not achieved as the targeted, addition of diuretic is done to the existing drug regime. Alan J. Zillich and others after reviewing a total of 59 clinical trials constituting 83 thiazide diuretic study arms demonstrated that treatment of thiazide-induced hypokalemia could lessen glucose intolerance and possibly the development of diabetes since hypokalemia has an effect on glucose intolerance. ${ }^{50}$ Study carried out by Gordanet $e t$ al show that in chronic hypokalemia patients there is a higher ratio of proinsulin to insulin secretion which leads to higher serum glucose concentrations since proinsulin is less active than insulin..$^{51}$ Among all hypertensive diabetic patients on thiazide diuretic therapy it is favorable to achieve $\mathrm{K} \geq 4.0 \mathrm{mmol} / \mathrm{L}$ concentration. This can be accomplished through the use of potassium-sparing diuretics and/or oral potassium supplementation since this will prevent hypokalemia.

\section{CURRENT TRENDS IN PRESCRIBING PATTERN OF THERAPEUTIC AGENTS FOR TREATMENT OF COEXISTENT TTDM AND HYPERTENSION}

For studying the current pattern of prescribingantihypertensive and oral hypoglycemic agents in treatment of coexistent TTDM and hypertension the patient survey was undertaken. Patients selected for the survey were of either sex having the mean age of $47 \mathrm{yrs}$. Mean HbA1c levels were found to be around $7.2 \%$ for all patients with average blood pressure of 140/90 $\mathrm{mm} \mathrm{Hg}$. The prescribing pattern of such around 100 patients was studied.

Data obtained from the survey showed that metformin was the highly prescribed oral hypoglycemic agent (97.93\%) compared to other oral hypoglycemics like sulphonylureas (63.91\%), DPP 4 Inhibitor (44.11\%). (Figure 1) These drugs were used either individually or in combination with each other to maintain blood plasma concentration. As amongst sulphonylureas choices were available glimepiride was found to be used by a wide number of patients $(88.7 \%)$ unlike others glipizide (1.61\%), gliclazide $(6.45 \%)$ and glibenclamide $(4.83 \%)$. (Figure 2) It was observed from the data that some patients were requiring some advance molecules for controling blood glucose levels like DPP-4 inhibitors. But their use was limited to $44 \%$ as compared to biguanides and sulphonylureas may be due to cost effectiveness of these conventional molecules. (Figure 


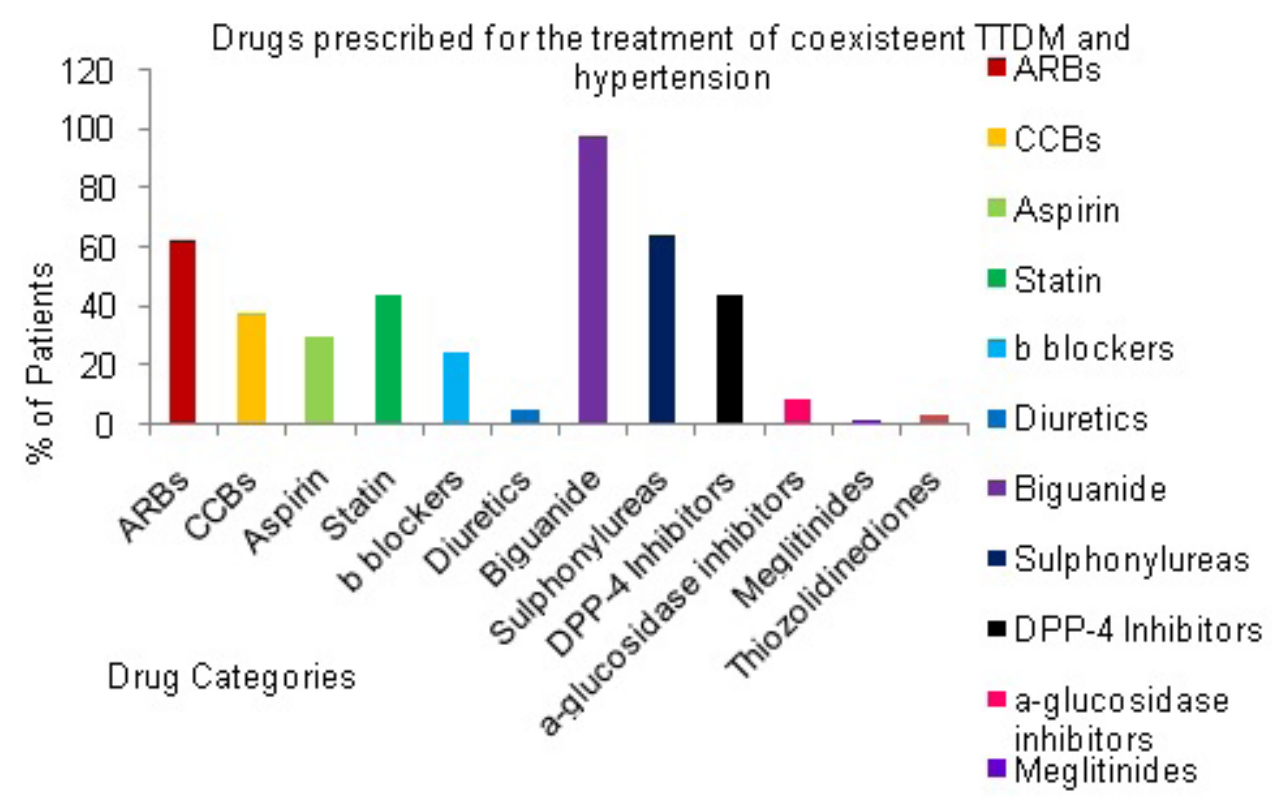

Figure 1: Drugs Prescribed For Treatment of Coexistent TTDM and Hypertension

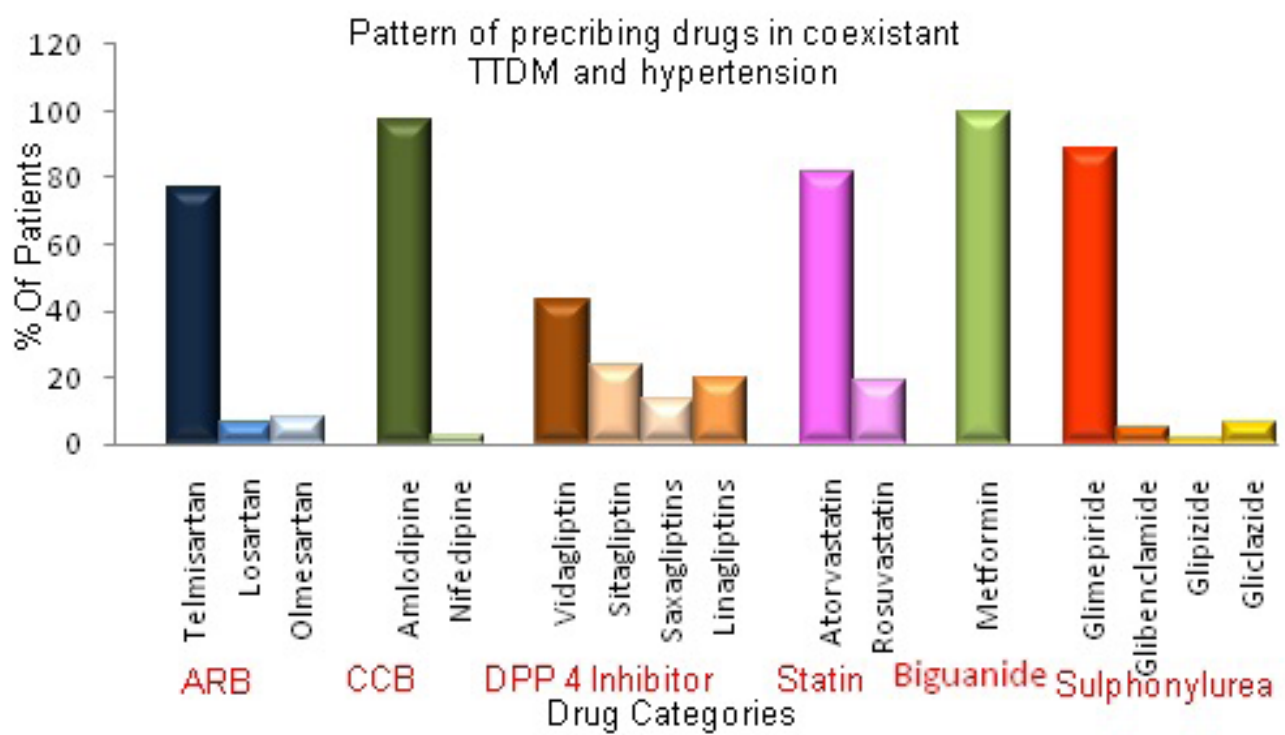

Figure 2: Pattern of prescribing drugs in coexistent TTDM and hypertension

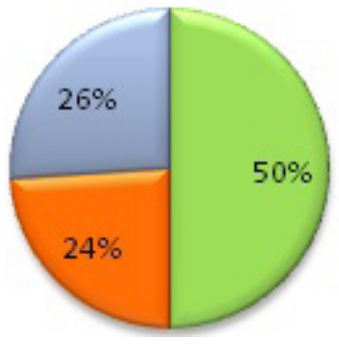

$\square$ Single Drug Dosage forms

$\square$ FDCsfor Hypertension

$\square$ FDCs for TTDM

Figure 3: Percentage of prescribing FDCs for hypertension and TTDM separately

1)Amongst DPP 4 Inhibitors vildagliptin (43.33\%) was widely used followed by sitagliptin $(23.33 \%)$.

It can be seen from the graph that for treating hypertension in TTDM, ARBs were the first and most accepted choice of physicians (62.88\%). (Figure 1) The renoprotective effect of ARBs independent of their blood pressure lowering effect made them treatment of choice. Telmisartan being immerging as 'cardiometabolic sartan' has been prescribed to high number of patients compared to other sartans.

Since the ADA has recommended and outcomes of several clinical trials have also supported the fact that $\geq 2$ agents are required to achieve the goal of 130/80 $\mathrm{mm} \mathrm{Hg}$ other antihypertensive agents like $\beta$ blockers and calcium channel blockers were also prescribed in $24.74 \%$ and $38.14 \%$ patients respectively. (Figure 1) It was observed during the survey that statins and aspirins were also prescribed in patients of coexistent TTDM 
and hypertension. We came across that $44.32 \%$ of people were on statin therapy and atorvastatin was a highly used statin. (Figure 1)

Patient survey has shown that almost $50 \%$ of dosage forms prescribed were individual drug dosage form. $26 \%$ Fixed Dose Combinations (FDC) of oral hypoglycemic agents were prescribed compared to usage of 24\% FDCs of antihypertensives. (Figure 3)

Thus, patient survey conducted supported the fact that combination therapy is indeed required for safe and effective treatment of coexistent TTDM and hypertension and it is well tolerated by all the patients.

\section{NEED OF FIXED DOSE COMBINATION THERAPY IN TREATMENT OF COEXISTENT TTDM AND HYPERTENSION}

Number of clinical trials conducted till date and patient survey conducted by us have conclusively demonstrated that polypharmacy or combination therapy is required to achieve targeted blood pressure $(130 / 80 \mathrm{~mm} \mathrm{Hg})$ and blood glucose levels in hypertensive diabetic patients. $\mathrm{ADA}$ and JNC 8 guidelines also suggest the use of $\geq 2$ agents as a first line therapy in such patients. In association with this hypertension, hyperglycemia, hyperlipidemia and renal disease are the modifiable risk factors for the coronary artery disease. Management of these risk factors with polypharmacy shows a $50 \%$ reduction in the occurrence of cardiovascular events. ${ }^{52}$ Similarly, to achieve additive or possibly synergistic glucose-lowering effects differently acting antidiabetic agents can be used in combination with the aim of enhancing therapeutic spectrum to combat with the progressive nature of the TTDM.

But noncompliance and lack of persistence with treatment is the major problem associated with the therapy of coexistent hypertension and TTDM. Contributing reasons for this poor adherence may be polypharmacy, complex drug regimen, increased pill burden,cost and the need of long term therapy. A study carried out by Marsha A. Raebel and others has shown that in patients with a newly ordered medication for hypertension, diabetes, or hyperlipidemia 7\% were found primarily non-adherent. ${ }^{53}$ Lower than $80 \%$ compliance was responsible for 1.39 times increased risk of undergoing a modification in the initially prescribed antihypertensive drug regimen. ${ }^{54}$ Similarly a study carried out by Ho PM et al demonstrated that significantly higher rates of morbidity and hospitalization, coupled with significantly higher $\mathrm{Hb} \mathrm{A} 1 \mathrm{C}, \mathrm{BP}$, and LDL-C levels were associated with $<80 \%$ adherence to their treatment regimen including oral antidiabetes agents, antihypertensive agents, and / or statin therapy compared with more adherent patients. Importantly, each 25\% improvement in adher- ence was associated with a reduction in A1C (0.05\%), systolic BP/ diastolic BP (SBP/DBP, 1.0/1.2 mm Hg), and LDL-C $(3.8 \mathrm{mg} / \mathrm{dL})$ that correlated with a significant $(\mathrm{P}<0.1)$ reduction in all-cause hospitalization and mortality ${ }^{55}$ This non adherence also leads to increased mortality and morbidity. ${ }^{56}$

Patients when switched to FDC from combination therapy shows improved adherence. A study carried out by Melikian $\mathrm{C}$ et.al in diabetic patients demonstrated that adherence to prescribed regimen was increased to $77 \%$ with the use of glyburide and metformin FDC compared to $54 \%$ when these agents were in 2 pills. ${ }^{57}$ Similarly it was found that compliance was 19\% greater in hypertensive patients taking lisinopril/HCTZ combination compared to drugs taken in 2 different tablets. ${ }^{58}$ Along with improving adherence FDC also lowers the cost involved in treatment of cardiovascular disease. ${ }^{59}$ Therefore use of FDCwill be one of the promising solution in treatment of coexistent TTDM and hypertension where patients have to take 4-5 tablets on an average per day and thus show poor compliance to therapy.

\section{FIXED DOSE COMBINATIONS}

CDSCO defines FDC as products containing two or more active ingredients used for a particular indication(s). ${ }^{60}$

Use of FDCs offer number of advantages such as decreasing pill burden, improved patient compliance, simplifying the complex dosage regimen, increased efficiency and safety corresponding to its pharmacological and therapeutic effects compared with monotherapy. But the major issue associated with use of FDC is its rationality. Irrational FDCs are usually responsible for adverse drug reactions. In case of pediatric nimesulide + paracetamol combination, addition of paracetamol doesn't lead to any additive or synergistic effect, instead it increases the risk of hepatotoxicity. When telmisartan + ramiprilcombination is used for treatment of hypertension, dual blockade of RAAS takes place which further worsens the symptoms. So this combination also exposes patient to unnecessary risk. NazaneenPourkavoos have thus categorized the FDC into three main categories and provides rational behind combining drugs $^{61}$

- Drug combination leading to the improvement of activity and/or tolerability: Combination of active ingredients having different mechanism of actions with the purpose of improved or broadened therapeutic effect having similar side effect profile compared to monotherapies. 
Example: olmesartan, hydrochlorthiazideand amlodipine combination for hypertension. ${ }^{62}$

- Drug combination leading to improvement in symptomatic and pharmacokinetic profile: Combining active ingredients with the aim of increasing intensity and duration of action of monotherapy by avoiding metabolic inactivation or elimination of the other active ingredient. Example: combination of sulphometoxazole and trimethoprim. ${ }^{63}$

- Drug combination leading to simplification of dosage regimen: With the aim of reducing pill burden and simplifying complex dosage regimen active ingredients are combined.

Example: Antitubercular FDC of Rifampicin, Ethambutal, Isoniazide and Pyrazinamide. ${ }^{64}$

\section{Challenges in development of FDC}

It is well understood now that the FDCs offer number of advantages but their formulation and development is indeed a very challenging task. During development of FDC formulator encounters several problems. To deal with these challenges prime importance should be given to

- Physical and chemical compatibility of all components in FDC.

- Pharmacokinetic and pharmacodynamic interactions of APIs (Active Pharmaceutical Ingredients).

- Alteration in the rate and extent of in-vitro dissolution and in-vivo bioavailability of APIs from FDC compared to free drug dosage form.

- Physical and compressional behavior of multiactive granulation.

- Volume of dosage form, especially in case of high dose combination.

Formulation of FDC into a conventional tablet or capsule rarely satisfies all these conditions. So there is a need to go with some modern approaches of formulation techniques.One of such approach is the use of multilayer tablet technology to avoid stability and compatibility problems. Micardis $\mathrm{HCT}^{\circledR}$ a bilayer tablet comprises a layer of telmisartan with alkalizing agent and a different hydrochlorthiazide layer to avoid degradation of hydrochlorthiazidein alkaline medium. ${ }^{65}$ At the same time this technology allows combination of sustained and immediate release layers in same dosage forms. Pulsatile drug delivery systems and multiphase capsule technology (tablet/powder blend or multiparticulate components coencapsulation) also serves as promising strategies for the development of FDC. Ecosprin Gold by USV is a coencapsulated product of enteric coated aspirin mini tablet and granules of clopidogrel and atorvastatin. ${ }^{66}$

FDCs also present a challenge in analysis also. When two components are present in the formulation then simultaneous equation method, absorbance ratio method, geometric correction method, two wavelength method, area under curve method and differential spectroscopy by using UV spectroscopy can be employed provided that two components suffice the basic requirements of each method. Such as for simultaneous equation method two drugs should absorb at absorption maxima of each other. But when more than two components are present UV spectroscopy methods have limitations to work effectively in quantification of individual components. Resolving the overlapped spectra of multi component mixtures (ternary or more) without prior separation of the constituent analytes becomes a difficult task. Chromatographic methods such as HPLC (Normal or reverse phase), HPTLC plays an important role.Many times hyphenated techniques such as LC-MS, LC-MSMS can also be used.

For development of FDC products Regulatory authorities have established several guidelines for industry. According to these guidelines it is mandatory to show that the FDC is bioequivalent to co-administration of the monotherapy provided that there is adequate safety and efficacy data for co-administration of the individual agents. For assessing the bioequivalence between FDC and monotherapy several methods are available. ${ }^{67}$

- Comparison of FDC and coadministration to show that $90 \%$ confidence interval (CI) of the geometric mean ratio (GMR) is between $80 \%$ and $125 \%$ for AUC and Cmax by employing crossover pharmacokinetic (PK) studies.

- Assurance of Pharmacodynamic (PD) Endpoint(s) achievement with therapeutic equivalence study.

- Combination of PK - PD studies. Establishment of equivalence between either AUC or Cmax is shown by using PK studies and PD study is to ensure either efficacy or safety is not compromised with the FDC

But BE studies present several challenges. Highly variable drugs, Metabolites and Non-linear Pharmacokinetics and drug-drug interactions are major ones ${ }^{68}$ Similarly food also affects the BE studies. BCS-based biowaiver are applicable to immediate-release FDC products in the USA when all actives in the FDC belong to BCS class I. Whereas in Europe the BCS-based biowaiver also includes BCS class III drugs and the excipients satisfying the criteria mentioned in EMA guidelines. Biowaiver 
of lower or intermediate strengths are applicable using the bracketing approach when BCS -based biowaiver is not applicable and if bioequivalence has been demonstrated at the highest and/or lowest strength. ${ }^{69}$

Though use of the FDC is proving to be a very effective solution for reducing the complications associated with therapy of coexistent TTDM and Hypertension, its formulation and Development is a tough task which requires detailed understanding of physicochemical and pharmacological properties of drugs, science of dosage form development and regulatory guidelines established by various authorities for marketing the developed FDC. It is also important to note that in the market there is not a single FDC available which will target simultaneously coexistent TTDM and hypertension. When hypertensive diabetic patients have to take on an average 5-6 tablets per day to manage their blood pressure and blood glucose levels, it leads to poor patient adherence due to complex regimen and pill burden which may further get translated into severe micro and macrovascular complications. But as findings from patient survey indicate that there is an absence of significant adverse interaction between multiple drugs used during therapy, these agents have the potential to get formulated into a single dosage form as FDC. Literature survey also suggests some antihypertensive and antidiabetic agents when given in combination leads to additive or synergistic effect which will help in reducing complications associ-

\section{REFERENCES}

1. Brunton L, Blumenthal D, Murri N, Dandan R, Knollmann B. Goodman \& Gilman's The Pharmacological Basis of Therapeutics. 12th ed. New York: McGraw-Hill; 2011.

2. American Diabetes Association. Standards of medical care in diabetes 2013. Diabetes Care 2013; 36(1): S11-66.

3. Shaw J, Sicree R, Zimmet P. Global estimates of the prevalence of Diabetes for 2010 and 2030. Diabetes Research and Clinical Practice 2010; 87(1): 4-14.

4. Marks J, Raskin P, Nefropatia E. Hypertension en la diabetes. Clin Med de NA. 1998; 4: 817-44.

5. Marre M, Berm G, Bouhanick B. Hypertension and diabetes mellitus. Biomedicine and Pharmacotherapeutics 1993; 47(2): 6 1-6.

6. Contreras F, Rivera M, Vasquez J. Diabetes and hypertension pathophysiology and therapeutics. Journal of Human Hypertension 2000; 14(1): S26-31.

7. KannelWB, McGee DL. Diabetes and cardiovascular risk factors: the Framingham study. Circulation 1979; 59(1): 8-13.

8. Epstein M , Sowers J. Diabetes mellitus and hypertension. Hypertension 1992; 19(5): 403- 18.

9. Tedesco M, Natale F, Di Salvo G, Caputo S, Capasso M, Calabro R. Effects of coexisting hypertension and Type 2 diabetes mellitus on arterial stiffness. Journal of Human Hypertension 2004; 18(7): 469-73.

10. Wong E, Backholer K, Gearon E, et. al. Diabetes and risk of physical disability in adults: A systematic review and meta-Analysis. Lancet Diabetes Endocrinology 2013; 1(2): 106-14.

11. Merel J, Luitse A, Biessels G, Guy E, Rutten H, Kappelle L. Diabetes, Hyperglycaemia, and acute ischaemic stroke. Lancet Neurology 2012; 11(3): 261-71.

12. Gleissner C, Galkina E,. Nadler J, Ley K. Mechanisms by which diabetes increases cardiovascular disease. Drug Discovery Today: Disease Mechanisms Cardiovascular Diseases 2007; 4(3): 131-41. ated with coexistence of TTDM and hypertension.Few to mention, the combination of telmisartan and aspirin ${ }^{70}$ combination of telmisartan and metformin ${ }^{71}$ and combination of pioglitazone and olmesartan. ${ }^{72}$ Development of FDC having antihypertensive and antidiabetic agents will be revolutionary in the treatment of coexistent TTDM and hypertension.

\section{CONCLUSION}

As the global epidemic of coexistent TTDM and hypertension is spreading with high speed, it is the right time to rethink over the formulation and development of medicines which will serve as a promising treatment for coexistent TTDM and hypertension. Due to complex regimen and increased pill burden these patients are less adherent to their treatment which then adversely affects their health. So the evelopment of FDC of antidiabetic and antihypertensive agents will be a very good answer to this question and therefore FDCs will undoubtedly play an important role in the treatment of coexistent TTDM and hypertension.

\section{ACKNOWLEDGEMENT}

We would like to express our profound gratitude towards Chellaram Diabetes Institute, Bavdhan, Pune and S.K.N. Hospital, Narhe, Pune for their help and support in conducting patient survey.

13. Today. Study group rapid rise in hypertension and nephropathy in youth with Type 2 Diabetes. Diabetes Care 2013; 36(6): 1735-41.

14. Benford M, Milligan G, Pike J, Anderson P, Piercy J, Fermer S. Fixed dose combination Antidiabetic Therapy: Real-World factors associated with prescribing choices and relationship with patient satisfaction and compliance. Advance Therapeutics 2012; 29(1): 26-40.

15. Chobanian AV. Impact of non adherence to antihypertensive therapy. Circulation 2009; 120(15): 1558-60.

16. International Diabetes Federation. Global guideline for Type 2 Diabetes; 2012.

17. Australian Government. National Health and Medical Research Council. National evidence based guidelines for the management of type 2 diabetes mellitus by Australian government, 2004.

18. Chobanian $\mathrm{AV}$, Bakris $\mathrm{G}$, Black $\mathrm{H}$, et. al. the national high blood pressure education program coordinating committee. The seventh report of the joint national committee on the prevention, detection, evaluation and treatment of high blood pressure. Hypertension 2003; 42(4): 1206-52.

19. Torre J, Bloomgarden Z, Dickey R, et.al. American association of clinical endocrinologists medical guidelines for clinical practicefor the diagnosis and treatment of hypertension. Endocrine Practice 2006; 12(2): 193-222.

20. Canadian Diabetes Association Clinical Practice Guidelines Expert Committee. Canadian Diabetes Association 2013 Clinical Practice Guidelines for the Prevention and Management of Diabetes in Canada. Can J Diabetes 2013;37(1):S1-S212.

21. Negro $\mathrm{R}$, Hassan $\mathrm{H}$. The effects of telmisartan and amlodipine on metabolic parameters and blood pressure in type 2 diabetic. Journal Of ReninAngiotensin-Aldosterone System 2006; 7(4): 243.

22. Connelly K, Kelly D, Langham R, Krum H, Gilbert R. Drug therapy for the cardiac complications of diabetes. Drug Discovery Today: Therapeutic Strategies 2004; 1(2): 195-200. 
23. Izuhara $Y$, Nangaku $M$, Inagi $R$, et.al. Renoprotective properties of angiotensin receptor blockers beyond blood pressure lowering. J Am SocNephrol. 2005; 16(12): 3631-41.

24. Yamagishia S, Takeuchi M. Telmisartan is a promising cardiometabolicsartan due to its unique ppar- $\gamma$-inducing property. Medical Hypotheses 2005; 64(3): 476-8.

25. Benson $\mathrm{S}$, Pershadsingh $\mathrm{H}, \mathrm{Ho} \mathrm{C}$, et.al. Identification of telmisartan as a unique angiotensin II receptor antagonist with selective ppar- $\delta$-modulating activity. Hypertension 2004; 43(5): 1-10.

26. Hongbo H, Yang D, Ma L, et.al. Telmisartan prevents weight gain and obesity through activation of peroxisome proliferator-activated receptor- $\delta$-dependent pathways. Hypertension 2010; 55(4): 869-79.

27. Suksomboon N, Poolsup N, Prasit T, et.al. Effects of losartan on renal and cardiovascular outcomes in patients with type 2 diabetes and nephropathy. $\mathrm{N}$ Engl J Med. 2001; 345(12): 861-9.

28. Lewis E, Hunsicker L, Clerk W, et.al. Renoprotective effect of the angiotensinreceptor antagonist irbesartan in patients with nephropathy due to type 2 diabetes. N Engl J Med. 2001; 345(12): 851-60.

29. Viberti $\mathrm{G}$, Wheeldon N. Microalbuminuria reduction with valsartan in patients with type 2 diabetes mellitus. Circulation 2002; 106(6): 672-8.

30. Haller $\mathrm{H}$, Ito $\mathrm{S}$, Izzo $\mathrm{J}$, et.al. Olmesartan for the delay or prevention of microalbuminuria in type 2 diabetes. N Engl J Med. 2011; 364(10): 907-17.

31. Salim Yusuf et al.The Ontarget Investigators. Telmisartan, ramipril, or both in patients at high risk for vascular events. N Engl J Med. 2008; 358(15): 1547-59.

32. Barnett A, Bain S, Bouter P, et.al. Angiotensin-receptor blockade versus converting-enzyme inhibition in type 2 diabetes and nephropathy. $\mathrm{N}$ Engl J Med. 2004; 351(19): 1952-61.

33. Lewis E, Lewis J. ACE inhibitors versus angiotensin receptor blockers in diabetic nephropathy: Is there a winner? J Am SocNephrol. 2004; 15(15): 1358-60.

34. Buse J, Ginsberg H, Bakris G, et.al. Primary prevention of cardiovascular diseases in people with diabetes mellitus. Diabetes Care 2007; 30(1): 162-72.

35. Kamari Y, Bitzur R, Cohen H, Shaish A, Harats D. Should all diabetic patients be treated with a statin? Diabets Care Nov 2009; 32(2): S378-83.

36. Hodel C. Myopathy and Rhabdomyolysiswith lipid-lowering drugs. Toxicology Letters 2002; 128(1): 159-68.

37. Awtry E, Loscalzo J. Aspirin. Circulation. 2000; 101(10): 1206-18

38. American Diabetes Association. Aspirin therapy in diabetes. Diabetes Care 2004; 27(1): S72-3.

39. Pignone $\mathrm{M}$, Alberts $\mathrm{M}$, Colwell $\mathrm{J}$, et.al. Aspirin for primary prevention of cardiovascular events in people with diabetes. Circulation 2010; 121(24): 2694-701.

40. Law E, Simpson S. Aspirin use rates in diabetes: A systematic review and cross-sectional study. Canadian Journal of Diabetes 2010; 34(3): 211- 7.

41. Mcgill J, Bakris G, Fonseca V, Raskin P, Messerli F, Phillips R. B-blocker use and diabetes symptom score: results from the GEMINI study diabetes. Obesity and Metabolism 2007; 9(3): 408-17.

42. Bakris G, Fonseca V, Katholi R, et. al. Metabolic effects of carvedilolvsmetoprolol in patients with type 2 diabetes mellitus and hypertension. JAMA. 2004; 292(18): 2227-36.

43. Cruickshank J. Beta-blockers and diabetes: The bad guys come good. Cardiovascular Drugs And Therapy 2002; 16(5): 457-70.

44. Majumdar S. Beta-blockers for the treatment of hypertension in patients with diabetes: Exploring the contraindication myth. Cardiovascular Drugs And Therapy 1999; 13(5): 435-9.

45. Cruickshank J. Beta-blockers continue to surprise us. European Heart Journal 2000; 21(5): 354-64.

46. Dunne F, Kendall M, Martin U. B-blockers in the management of hypertension in patients with type 2 diabetes mellitus. Drugs 2001; 61(4): 429-35.

47. Masuda T, Ogura M, Moriya T. Beneficial effects of L- and N-type calcium channel blocker on glucose and lipid metabolism and renal function in patients with hypertension and type II diabetes mellitus. Cardiovascular Therapeutics 2011; 29(1): 2946-53.

48. Yousef W, Omar A, Morsy M. The role of calcium channel blockers in the treatment of diabetic nephropathy. Int J Diabetes \& Metabol. 2005; 13(2): 68-75.
49. Toto R, Tian M, Fakouhi M, Champion A, Bacher P. Effects of calcium channel blockers on proteinuria in patients with diabetic nephropathy. J Clin Hypertens (Greenwich). 2008; 10(10): 761-9.

50. Zillich A, Garg J, Basu S, Bakris G, Carter B. Thiazide diuretics, potassium, and the development of diabetes: A quantitative review. Hypertens. 2006; 48(2): 219-24

51. Gorden P, Sherman B, Simopoulos A. Glucose intolerance with hypokalemia: an increased proportion of circulating proinsulin-like component. J Clin Endocrinol Metab. 1972; 34(1): 235-40.

52. Gaede P, Vedel P, Larsen N, et.al. Multifactorial intervention and cardiovascular disease in patients with type 2 diabetes. N Engl J Med. 2003; 348(5): 383-93.

53. Raebel M, Ellis J, Carroll N, et.al. Characteristics of patients with primary nonadherence to medications for hypertension, diabetes, and lipid disorders. $\mathrm{J}$ Gen Intern Med. 2011; 27(1): 57-64.

54. Boris L, Van W, Klungel $\mathrm{O}$. The association between compliance with antihypertensive drugs and modification of antihypertensive drug regimen. Journal of Hypertension 2004; 22(9): 1831-7.

55. Ho P, Rumsfeld J, Masoudi F, et.al. Effect of medication nonadherenceon hospitalization and mortality among patients with diabetes mellitus. Arch Intern Med. 2006; 166(17): 1836-41.

56. Bailey $\mathrm{C}$, Kodack M. Patient adherence to medication requirements for therapy of type 2 diabetes. Int J Clin Pract. 2011; 65(3): 314-22.

57. Melikian C, White T, Vanderplas A, Dezii C, Chang E. Adherence to ora antidiabetic therapy in a managed care organization: a comparison of monotherapy, combination therapy, and fixed-dose combination therapy. ClinTher. 2002; 24(3): 460-7.

58. Dezii C. A retrospective study of persistence with single-pill combination therapy vs. Concurrent two-pilltherapy in patients with hypertension. Managed Care 2000; 9(9): S2-6

59. Taylor A, Shoheiber O. Adherence to antihypertensive therapy with fixeddose amlodipine besylate/benazepril $\mathrm{HCl}$ versus comparable componentbased therapy. Congest Heart Fail. 2003; 9(6): 324-32.

60. Guidance for Industry on Fixed Dose Combination By CDSCO, Directorate General of Health Services. Ministry of Health \& Family Welfare Govt. of India; April 2010.

61. Pourkavoos N. Unique risks, benefits, and challenges of developing drugdrug combination products in a pharmaceutical industrial setting. Comb Prod Ther. 2012; 2(1): 1-31.

62. Chrysant S. Triple-drug, fixed-dose combinations for the treatment of hypertension: Focus on olmesartan/amlodipine/hydrochlorothiazide combination. Drugs Today (Barc) 2011; 47(3): 197-206.

63. Huovinen P. Resistance to trimethoprim-sulfamethoxazole. Clinical Infectious Diseases 2001; 32(11): 1608-14.

64. Blomberg B, Spinaci S, Fourie B, Laing R. The rationale for recommending fixed-dose combination tablets for treatment of tuberculosis. Bulletin of The World Health Organization 2001; 79(1): 61-8.

65. Product Information Leaflet For Micardis HCT®

66. Product Information Leaflet For Eccosprin Gold®

67. Mastan S, Latha T, Ajay S. The basic regulatory considerations and prospects for conducting bioavailability/bioequivalence (BA/BE) studies-An overview. Comp Eff Res. 2011; 1 (issue missing?): 1-25.

68. Jackson A, Robbie G, Marroum P. Metabolites and bioequivalence: Past and present. Clin Pharmacokinet. 2004; 43(10): 655-72.

69. Mitra $\mathrm{A}, \mathrm{Wu} \mathrm{Y}$. Challenges and opportunities in achieving bioequivalence for fixed-dose combination products. The AAPS Journal 2012; 14 (3): 646-55

70. Mulay S, Gaikwad A, Tikoo K. Combination of aspirin with telmisartan suppresses the augmented tgf $\beta /$ smad signaling during the development of streptozotocin-induced type I diabetic nephropathy. Chemico-Biological Interactions 2010; 185(2): 137-47.

71. Goyal A, Goyal R, Sharma P, Malik Z. Beneficial effect of combination of metformin and telmisartan on high fat diet-induced obesity in wistar rats Asian J Pharm Clin Res. 2011; 4(4): 106-11.

72. Sengupta P, Nandi U, Pal T. Development of safety profile evaluating pharmacokinetics, pharmacodynamics and toxicity of a combination of pioglitazone and olmesartanmedoxomilinwistar albino rats. Regulatory Toxicology and Pharmacology 2012; 62(1): 7-15. 